\title{
Double Test: A Simplified Alternative for Cervical Cancer Screening in Low Resource Countries
}

\author{
Atef M.Darwish ${ }^{1 * *}$ MD, PhD, Howieda Fouly ${ }^{1} *$ MSN, PhD, Sahar Naguib ${ }^{1 *}$, PhD \\ Marilyn Stringer ${ }^{2 * *}$, Dalal Eshra ${ }^{3 * * *}$, PhD, ${ }^{4}$ Mohamed Galal\$ PhD \\ ${ }^{1 *}$ Departments of Obstetrics and Gynecology, Woman's Health University Center, Assiut University, Egypt \\ ${ }^{1 *}$ Obstetrics \& Gynecology Nursing, Faculty of Nursing, Assiut University, Egypt \\ ${ }^{2 * *}$ School of Nursing, University of Pennsylvania, Philadelphia, USA \\ $3^{3 * * *}$ Menoufia University. Menoufia Egypt \\ ${ }^{4}$ Department of Pathology, Facult y of Medicine, Assiut University, Assiut, Egypt
}

\begin{abstract}
:
Objectives: To test if combining positive results of sequential magnified naked-eye examination (MNEE) and visual inspection of cervix after application of acetic acid (VIA) would increase diagnostic indices for precancerous and cancerous cervical lesions in comparison to the gold standard positive Pap test in a developing country set up. A secondary objective was to evaluate the effectiveness of nurse training and patient acceptance of this screening approach.
\end{abstract}

Design: An analytic cross-sectional research design

Setting: Gynecology and infertility out-patient clinics of the departments of Obstetrics and Gynecology, Woman's Health University hospital, Assiut University, Egypt.

Materials and methods: A total of 445 non-pregnant women aged between 17 and 44 years were counseled and entered this study. They were subjected to MNEE after cleaning of the cervix with $0.9 \%$ saline, exfoliative cytology and lastly VIA. Positive results were evaluated and managed accordingly. Colposcopy with or without subsequent cervical biopsy were done in all positive cases of MNEE, VIA and Pap smear. Main outcome measures included diagnostic accuracy of VIA alone versus VIA and MNEE as compared to cytology.

Results: This study comprised 445 non pregnant women in the reproductive age. The main complaint was abnormal vaginal discharge in 403 cases $(89.6 \%)$. MNEE of the cervix indicated that 338 women $(75.1 \%)$ and 112 women $(24.9 \%)$ had healthy and unhealthy-looking cervices respectively. Pap smear sampling was negative for premalignant or malignancy in 377 cases (83.8\%).VIA test findings were positive in 77 cases (17.3\% ) and negative in 368 cases $(82.6 \%)$ All diagnostic indices increased significantly after combing MNEE and VIA positive results except negative predicative value if compared to VIA positive results alone as an alternative to the standard Pap smear. Nurses proved high level of skill in performing cervical cytology, MNEE and VIA as proved by lower rate of unsatisfactory results. Patient acceptability was very high after proper counseling.

Conclusions: Sequential MNEE and VIA would improve most of diagnostic indices as an alternative diagnostic tool to the gold standard Pap smearing for detection of abnormal precancerous and cancerous cervical lesions. This simplified cheap approach with high percentage of patient acceptability would help expanding screening programs in countries were Pap smear is poorly available. Being performed by doctors as well as trained nurses on one-stop base is a clear advantage. More large sample sized studies on the cost-effectiveness of this approach versus colposcopically-guided positive histopathology are recommended before its universal spread.

Key words : Cervical Cancer, Magnified Visual Inspection, Acetic Acid, MNEE, VIA, Cytology.

\section{INTRODUCTION}

Unfortunately, most of women who develop invasive cervical cancer are discovered in developing countries. Screening programs utilizing Pap test are considered the optimum strategy to reduce cervical cancer incidence (1-3). Alternative approaches have been evaluated including unaided naked eye examination (UNEE), automated Pap

1 ***orresponding Author: atef_darwish@yahoo.com 
screening, visual inspection with acetic acid (VIA) testing, Schiller's iodine test, human papillomavirus virus testing and the polar probe testing $(4,5)$ Some of these tests are not suitable for low-resource countries $(6)$ due to financial or man power restrictions. In a recent large-sample sized study (7), our team succeeded to achieve excellent sensitivity on UNEE in comparison to Pap smear. Nevertheless, a common pitfall of all these diagnostic tools including Pap test is low specificity for diagnosis of precancerous cervical lesions. Combination of more than one diagnostic tool is a recent attractive approach aiming at increasing efficacy of screening programs. In a recent study, combination of VIA and Schiller's iodine test achieved excellent sensitivity but less specificity than Pap test (8). Another recent trial used combination of polymerase chain reaction technique in real time (Cobas 4800 device) with liquid cytology (9). This study aims to test if combining positive results of sequential magnified naked-eye examination (MNEE) and visual inspection of cervix after application of acetic acid (VIA) as a double screening tool for cervical cancer would increase diagnostic indices of diagnosing precancerous and cancerous cervical lesions in comparison to Pap test in a developing country set up. A secondary objective was to evaluate the effectiveness of nurse training and patient acceptance of this diagnostic approach.

\section{Patients ANd Methods}

The prospective cross sectional study included non-pregnant women attending out-patient clinics at Woman's Health University Hospital, Assiut, Egypt in the period from July 2010 to August 2012. This study was approved by the Institutional Review Board (IRB) of the Faculties of Medicine and Nursing. All women gave a written consent to participate in this study after proper explanation of the procedure and the subsequent steps of management. Exclusion criteria were women with excessive bleeding, women who never married, non-reproductive periods (postmenopausal or prepubertal periods) or non-Egyptian women.

\section{SAMPLE SIZE ESTIMATION}

A sample size of 445 subjects was used to provide information via Pap smear, MNEE and VIA to achieve $80 \%$ power to detect an odds ratio of 1.989 using a two-sided McNemar test with significance level of 0.05 . The odds ratio was equivalent to a difference between the two paired proportions of 0.056 which occurs when the proportion of subjects with -VIA and MNEE/+Pap is 0.113 and the proportion of subjects with +VIA and MNEE/-Pap was 0.057 . The overall proportion of discordant pairs was assumed to be 0.170 . Therefore, a sample size of 445 was adequate to answer the study questions. $(9,10)$.

\section{QUESTIONNAIRE}

The provider (HF) was a highly educated nurse, who studied basic anatomy and physiology of the cervix and received few weeks of training by the unit's director (AD) on how to perform MNEE and VIA screening test. A thorough history was taken with special emphasis on age of the patient, age at and duration of marriage, parity, menstrual pattern and date of the cycle, contraceptive history; type and duration, contact bleeding or dyspareunia, vaginal discharge, color, odor, and associated symptoms.

\section{MANEUVERS}

In the lithotomy position, a non-lubricated bivalve vaginal speculum was inserted into the vagina. The cervix was cleaned from any secretions or blood by a piece of cotton embedded in $0.9 \%$ saline. A thorough magnified nakedeye examination (MNEE) of the cervix utilizing a simple hand lens which magnifies twice was done to detect any apparent lesions particularly abnormal vessels with abrupt ending. If a cervical lesion was seen it was recorded and its site was marked on a special diagram. Cervical Pap smears were obtained using the long tip of Ayre's spatula which was placed in the endocervical canal with the proximal bulge resting on the ectocervix. The spatula was carefully rotated around the cervix so that a representative sample of the whole cervix was obtained. An additional endocevical sample was obtained by placing a cytobrush in the endocervical canal and gently rotated through 360 degrees as previously described (11). The samples were then immediately plated on a slide, fixed by immersing the slide in 95\% ethyl alcohol fixative for 15-20 minutes and sent to the pathologist who stained the smear by modified Papanicolaou stain using hand staining procedure. Each smear was screened using Modified Bethesda system terminology and checked for cytological evidence of inflammation with or without cellular atypia, dysplasia or carcinoma. Thereafter, the cervix was painted by the provider (H F) with acetic acid 5\% and properly examined. Abnormal areas on the ectocervix stained with acetic acid will turn into white color. Likewise, cervical ectopy will turn into white color. All these acetowhite areas (VIA positive) particularly those with very sharp borders were landmarked in the report to be send to the coloposcopist (AD). If the cervix didn't stain with acetic acid, the test was considered VIA negative. All these steps were performed by the nurse under strict supervision of the gynecologist (AD). The reports of MNEE, cytology, and VIA were evaluated by A.D. who decided further management plan 
accordingly. Positive cases with abnormal Pap Smear or visible cervical lesions by MNEE or positive VIA were scheduled for colposcopic examination using Karl Kaps Colposcope model (SOM 52), Germany. Using a punch biopsy forceps, a colposcopically-guided biopsy was performed by A.D. in cases of abnormal colposcopic examination including aceto-while areas with mosaic or punctuation, abnormal vasculature as seen with green filter or suspicious nodules. Histologically proved precancerous cervical lesions (CIN II, III or microinvasion) were treated with large loop excision (LLETZ) of the cervix or extrafascial hysterectomy according to the age and parity of patients with meticulous follow-up. Cases with CIN I were followed up with cytology and colposcopy. If persisted or advanced, LLETZ was performed later on. Cases with proved invasive cervical cancer were referred to the oncology team at our institution. Knowledge of women regarding screening programs and cervical cancer were simultaneously evaluated by the nursing team.

\section{STATISTICAL ANALYSIS}

Data entry was done using Epi-Info 6.04 computer software package, while statistical analysis was done using SPSS 13.0 for windows statistical software packages. Quality control was done at the stages of coding and data entry. Data were presented using descriptive statistics in the form of frequencies and percentages for qualitative variables, and ranges, means and standard deviations, medians and quartiles for quantitative variables in box plots. The comparability of baseline characteristics according to outcome was ascertained by Student's t test (unpaired t test) for continuous variables and Mann Whitney $\mathrm{U}$ test when appropriate, and $\mathrm{X}^{2}$ test (Chi square test) for categorical variables. To evaluate the univariate relation between variables, Pearson's correlation coefficient was calculated. Values were considered significant if $\mathrm{p}$ value $<0.05$. Diagnostic indices for VIA alone or sequential MNEE and VIA were calculated. The diagnostic rates including sensitivity, specificity, positive and negative predictive values were determined. The diagnostic accuracy for each approach was calculated using the formula:

$$
\text { Diagnostic accuracy }=\frac{(T P+T M)}{(T P+T N+F P+F N)} \times 100
$$

where TP: true positive, TN: true negative, FP: false positive, FN: false negative.

\section{RESULTS}

This study comprised 445 non pregnant women in the reproductive age group who agreed to participate in this study $(100 \%)$. Their mean age was 34.9(SD \pm 5.9 years). Regarding residence, the majority (400 cases, $88.9 \%$ ) were from rural areas, while only 50 cases $(11.1 \%)$ lived in urban areas. Regarding the women's education, $289(64.2 \%)$ were illiterate, compared to 20 (4.4\%) were highly educated (figure 1). Table (1) shows the distribution of the participating women by their marriage characteristics. All women had a single partner (no previous marriage). The main complaint in most cases was abnormal vaginal discharge (89.6\%) while other complaints are shown in table (2). MNEE of the cervix indicated that 338 women(75.1\%) and 112 women (24.9\%) had healthy and unhealthylooking cervices respectively as shown in table (3). Cervical smear was satisfactory in most cases $99.3 \%$ ( $\mathrm{n}=440$ ). Unsatisfactory samples were described by the pathologist as blood-tinged specimens or poor smearing of specimens. Forty-six subjects (10.3\%) had atypical smears associated with inflammatory smear. Negative smears were defined as normal with no evidence of malignancy as shown in table (4). VIA test findings were positive in 77 cases ( $17.3 \%$ ) and negative in 368 cases (82.6\%). The overall accuracy (diagnostic accuracy) of the screening index (VIA alone or combined MNEE and VIA) against the reference (Cytology) was estimated by the proportion of $(\mathrm{TP}+\mathrm{TN}) / \mathrm{N}$ where $\mathrm{N}$ equals the total number of patients being analyzed $(\mathrm{N}=445)$ as demonstrated in table (5). All diagnostic indices increased significantly on sequential MNEE and VIA results except NPP if compared to VIA alone as an alternative to the standard Pap smear. Colposcopy as well as biopsy results will be discussed in a coming separate study.

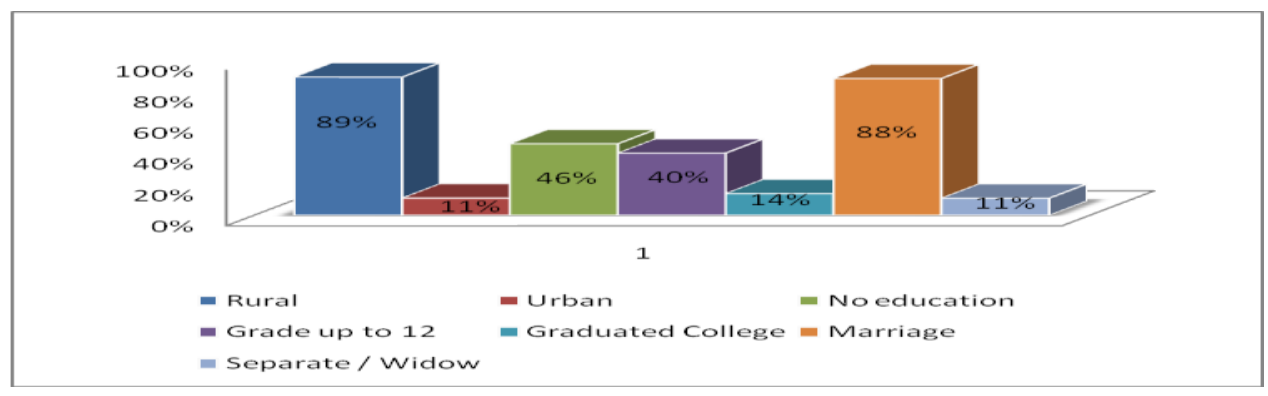

Fig1. Sociodemographic characteristics of the studied population. 
American Research Journal of Nursing, Volume 1, Issue 2, June-2015

ISSN 2379-2922

Table1. Marital status $(N=450)$

\begin{tabular}{|l|l|l|}
\hline Marriage characteristics & Number & Percentage (\%) \\
\hline Marital status & & \\
\hline Married & 417 & 92.7 \\
\hline Widow & 26 & 5.8 \\
\hline Separated & 7 & 1.5 \\
\hline Age at Marriage & & \\
\hline$<18$ & 178 & 40 \\
\hline $18-25$ & 230 & 51.7 \\
\hline$>25$ & 37 & 8.3 \\
\hline Duration of marriage & & \\
\hline$\leq 6$ years & 126 & 28 \\
\hline $7-12$ & 123 & 27.3 \\
\hline $13-18$ & 97 & 21.6 \\
\hline $19-24$ & 52 & 11.5 \\
\hline $25-31$ & 40 & 8.9 \\
\hline$\geq 32$ & 12 & 2.7 \\
\hline Total & $\mathbf{4 5 0}$ & $\mathbf{1 0 0}$ \\
\hline
\end{tabular}

Table2. Main gynecological complaints

\begin{tabular}{|l|c|c|c|c|}
\hline & $\frac{Y e s}{N}$ & $\%$ & $\frac{N o}{N}$ & $\%$ \\
\hline Excessive vaginal discharges & 403 & 89.6 & 47 & 10.4 \\
\hline Chronic pelvic pain & 223 & 51.8 & 217 & 48.2 \\
\hline Anogenitalia ulcers & 143 & 31.8 & 307 & 68.2 \\
\hline Lower abdominal pain & 351 & 78.0 & 99 & 22.0 \\
\hline Dyspareunia & 298 & 66.2 & 152 & 33.8 \\
\hline Postcoital bleeding & 88 & 19.6 & 362 & 80.4 \\
\hline Intermenstrual bleeding & 116 & 25.8 & 334 & 74.2 \\
\hline Low backache & 356 & 79.1 & 94 & 20.9 \\
\hline Vaginal dryness & 280 & 62.2 & 170 & 37.8 \\
\hline
\end{tabular}

Table3. MNEE findings*.

\begin{tabular}{|l|c|c|}
\hline Visual Examination of the cervix & Frequency & Percent \\
\hline Normal healthy cervix & 338 & 75.1 \\
\hline Cervical polyp. & 60 & 13.3 \\
\hline Nabothian follicles & 67 & 14.9 \\
\hline Cervicitis & 137 & 30.4 \\
\hline Leukoplakia & 14 & 3.1 \\
\hline Condyloma & 7 & 1.6 \\
\hline Abnormal growth & 3 & 0.6 \\
\hline
\end{tabular}

More than one abnormal finding in the same case.

Table4. Cytologic findings

\begin{tabular}{|l|c|c|}
\hline Pap smear findings & Frequency $\mathrm{n}=$ & Percent \% \\
\hline Negative & 377 & 83.8 \\
\hline Atypical cells with inflammatory smear & 46 & 10.2 \\
\hline SIL Low grade & 18 & 4.0 \\
\hline SIL High grade & 3 & 0.7 \\
\hline Squamous cell cancer & 1 & 0.2 \\
\hline Unsatisfactory sample & 5 & 1.1 \\
\hline Total & $\mathbf{4 5 0}$ & $\mathbf{1 0 0 \%}$ \\
\hline
\end{tabular}


American Research Journal of Nursing, Volume 1, Issue 2, June-2015

ISSN 2379-2922

Table5. The diagnostic indices of screening tests VIA alone or combined MNEE and VIA in comparison to cytology.

\begin{tabular}{|l|c|c|}
\hline Diagnostic indices & VIA Vs. Pap smear & VIA and MNEE Vs. Pap smear \\
\hline Sensitivity (\%) & 59 & 73.8 \\
\hline Specificity (\%) & 85.6 & $96.7 \%$ \\
\hline PPV (\%) & 19.4 & $84.1 \%$ \\
\hline NPV (\%) & 97.2 & $93.8 \%$ \\
\hline Diagnostic Accuracy & $75 \%$ & $87.2 \%$ \\
\hline
\end{tabular}

VIII. DISCUSSION

Developing counties are generally burdened by inadequate manpower, insufficient funds and particularly by non compliance of the patients and it is better to search for a restricted screening program focusing mainly on the high risk group of women including women with suspicious cervix, repeated genital infection and associated genital or breast malignancies (12). The same concept was suggested by many authors who stated that, the generally accepted and highly successful screening regimen of repeated cytological smears of the cervix is inappropriate for implementing in developing countries; subsequently a more simplified and less expensive approach is highly necessary (13). A number of alternative methods for regular Pap smear have been suggested. A limitation of the numbers of smears per life is favored by WHO, it has been estimated that three smears at appropriate ages can effectively reduce the incidence of cervical cancer by $46 \%$, This hypothesis however has not been adequately tested (14). According to UNEE, women are usually categorized into two groups, women with healthy looking cervix and women with suspicious cervix. The term suspicious is used by many authors to describe the unhealthy looking cervix $(15,16)$. Nevertheless, we can't ignore the valuable role of both MNEE and VIA in localizing the abnormal lesion on the cervix that would be easily biopsied at colopscopic examination. The results of UNEE were superior to VIA which has a sensitivity of $71 \%$ and specificity of $74 \%$ (7). From studies which were done on VIA, it is demonstrated that VIA has high sensitivity for detecting cervical intraepithelial neoplasia ( CIN) and cervical cancer, but on the other hand it has low specificity $(5,17)$. Schiller's iodine test (SIT) is done by painting the cervix with iodine so that high glycogen cells i.e. squamous cells would turn brown while low glycogen cells e.g. columnar cells, metaplastic cells or CIN will not be pigmented with the dye (18). It is well-documented that UNEE is a basic and logic step prior to VIA that is done in all cases all over the world. However, all studies on VIA rely on positive findings after acetic acid application and omit UNEE findings. It should be known that not all positive cases with UNEE are essentially positive on VIA. Seeing cervical cysts like Nabothian follicles or other well epithelialized lesions, strawberry cervix associated with cervicitis or tiny excessive or abnormal suspicious vasculature seen with UNEE are usually negative with VIA. What's new in this study is the use of a simple hand lens to magnify the cervix. Moreover, respecting each positive finding utilizing either tool and comparing collective diagnostic indices of MNEE and VIA to VIA alone is a novel idea. Using sequential UNEE and VIA as an alternative method of cervical cancer screening to Pap test is characterized by being fast, simple, and easily learned with appropriate training by non-physician providers such as nurses. Other international researchers have demonstrated similar findings indicative of nurses safely expanding their scope of practice to include MNEE and VIA screening as demonstrated in the initial pioneering studies $(19,20)$. It seems logical to consider colposcopically-guided cervical biopsy positive results the gold standard for this study. Unfortunately, this was not applicable as colposcopy was only done for positive cases with Pap smear. Selection of the Pap smear as the reference test for VIA or sequential MNEE and VIA was based on two reasons. Firstly, developed countries depend on Pap smears as a screening test for cervical cancer. Secondly, the ability to compare our study findings to similar previous studies (21-24) required comparison to Pap test as it was the gold standard in all these studies. In the current study, the specificity of VIA alone compared to Pap smear screening was $85.6 \%$. Some researchers reported higher specificity rates $(25,26)$ or lower rates (27-30) with high negative predictive values (NPV). These findings may vary due to the differences in incidence of cervical cancer between populations. In Egypt, based on WHO report in 2010, the annual crude incidence rate was 1.3/100000 women/year whereas it was 15.8/100000 women worldwide (22). Another explanation of variable results is the inclusion or exclusion of atypical cells differently. In one study (26), there were $24.8 \%$ ASCUS, followed by $3.8 \%$ of low grade and $0.7 \%$ of high-grade lesions. Akinola et al (31), reported similar results of inflammation or ASCUS, followed by LGSIL and HGSIL. Both studies found lack of cytology accuracy was related to a high percentage of ASCUS. Our findings indicated similarities in grading which ASCUS the biggest percentage $10.2 \%$ followed by $4 \%$ of low grade and $(0.9 \%)$ high grade. From studies which were done on VIA, it is demonstrated that VIA has high sensitivity for detecting cervical intraepithelial neoplasia (CIN) and cervical cancer, but on the other hand it has low specificity $(15,16)$. As seen in table 5 , all diagnostic indices improved if we 


\section{American Research Journal of Nursing, Volume 1, Issue 2, June-2015 ISSN 2379-2922}

combined the results of MNEE and VIA except lower NPP than VIA alone. This interesting finding was not clearly addressed in previous studies as most papers usually focus on one diagnostic aid. Due to our previous big samplesized study of 3500 cases using UNEE as an alternative to cytology which achieved a high sensitivity of $91.16 \%$ we were encouraged to construct this study to increase the diagnostic accuracy by combining MNEE and VIA in comparison to Pap smearing. The main pitfall of our previous study on UNEE was lower specificity if compared to cytology $(91.16 \%, 100 \%)$. This was another indication of constructing this study. Omitting colposcopically-guided biopsy results in this study and considering Pap smear as a gold standard reference is a clear weak point. The problem was heterogeneity of positive cases on MNEE, VIA, Pap smear and positive biopsy that made statistical analysis of the results impossible. To rely on histopathologic diagnosis as a gold standard, a very big large sample sized further study may be required. From this study, it is concluded that sequential MNEE and VIA would improve the diagnostic accuracy as an alternative to Pap smearing for detection of abnormal precancerous and cancerous cervical lesions. This simplified cheap approach would help expanding screening programs in countries were Pap smear is poorly available. Being performed by doctors as well as trained nurses as a one-stop diagnostic tool is a clear advantage. More large sample sized studies on the cost-effectiveness of this approach versus colposcopicallyguided positive histopathology are recommended before its universal spread.

\section{REFERENCES}

[1] Miller AB, Naseer S. Fann S. Brand up ukonow A. Rehman, Conje H, report on consensus on cervical cancer screening and management, Int. J cancer 2000;86:440 447.

[2] Shiffman $\mathrm{MH}$, recent progress in defining the epidemiology of human papillomavirus infection and cervical neoplasia. J Nat Can Inst $1992 ; 84: 394-397$.

[3] Benedet JL. Progress in gynecologic cancer detection and treatment. inter .J.Gyn Oncol 2000; 70: 135-147.

[4] Goel A, Gandhi G, Batra S, Bhambhani S, Zutshi V, Sachdeva P. Visual inspection of the cervix with acetic acid for cervical intraepithelial lesions. Int J Gynaecol

[5] Obstet. 2005;88(1):25-30.

[6] Sankaranarayanan R., Wesley R., Somanathan T., Dhakad N., Shyamalakumary B., Sreedevi A. N., et al. Visual inspection of the uterine cervix after the application of acetic acid in the detection of cervical carcinoma and its precursors. Cancer 1998; 83, 2150-15.

[7] Sankaranarayanan R, Budukh AM, Rajkumar R. Effective screening programms for cervical cancer in lowand middle-income developing countries. Bulletin of the World Health Organization 2001;79:954-962.

[8] Darwish A M, Abdullah SA, Zahran K S, Abdel-Fattah N. Reliability of naked eye examination for the diagnosis of benign cervical lesions. J Low Genit Tract Dis. 2013 Apr;17(2):182-6.

[9] Consul S, Agrawal A, Sharma H, Bansal A, Gutch M, Jain N. Comparative study of effectiveness of Pap smear versus visual inspection with acetic acid and visual inspection with Lugol's iodine for mass screening of premalignant and malignant lesion of cervix. Indian J Med Paediatr Oncol. 2012 Jul;33(3):161-5.

[10] Kogan EA, Faĭzullina NM, Demura TA, Kozachenko AV, Pavlenko OA, Permiakova OA. The optimal screening of cervix cancer: combination of polymerase chain reaction technique in real time (Cobas 4800 device) with liquid cytology. Klin Lab Diagn. 2012 Dec;(12):18-20.

[11] Schork, M. A., William, G. W. in Statistics - Simulation and Computation. 1980;9, 4, 349 - 357.

[12] Machin D, Campbell M, Fayers P, Pinol A. Sample Size. Tables for Clinical Studies. Second Ed. Blackwell Science 1997; IBSN 0-86542-870-0 p. 73-74

[13] World Health Organization. Cervical cancer screening in developing countries: report of a WHO consultation. World Health Organization 2002.

[14] Stjersward J. plotting a new course for cervical cancer screening in developing countries. WHO forum 1987;8 : $42-52$.

[15] WHO meeting. Control of cancer of the cervix uteri. Bull world Health Organ1997;64:607-618

[16] Meisels, A. Hormonal cytology. In: Compendium on diagnostic cytology. 5th Ed. Edited by Keebler, C.M. Illinois. Chicago.1983; p. 250.

[17] Darwish A, Gadallah H. One-step management of cervical lesions. Int J Gynaecol Obstet. 1998;61(3):261-7.

[18] Megevand E, Denny L, Dehaeck K, Soeters R, Bloch B. Acetic acid visualization of the cervix: an alternative to cytologic screening. Obstet Gynecol 1996; 88(3): 383-386. 
[19] Qureshi S, Das V, Zahra F. Evaluation of visual inspection with acetic acid and Lugol's iodine as cervical cancer screening tools in a low-resource setting. Trop Doct. 2010;40(1):9-12.

[20] University of Zimbabwe/JHPIEGO Cervical Cancer Project Visual inspection with acetic acid for cervicalcancer screening: test qualities in a primary-care setting. Lancet. 1999;353:869-73.

[21] Sarian LO, Derchain SF, Naud P, Roteli-Martins C, Longatto-Filho A, Tatti, S, et al. Evaluation of visual inspection with acetic acid (VIA), Lugol's iodine (VILI), cervical cytology and HPV testing as cervical screening tools in Latin America. Journal of Medical Screening 2005; 12(3),142-149.

[22] Muwonge R., Manuel M., Antonio P., Filipe C., Dumas J.B., Frank M. R., Sankaranarayanan R. Visual screening for early detection of cervical neoplasia in Angola. Int J Gynecol Obstet 2010; 111, 68-72.

[23] WHO/ICO Information Centre on HPV and Cervical Cancer. (HPV Information Centre). Human Papillomavirus and Related Cancers in Egypt. Summary Report.2010 Available at http// www.who.int/hpvcenter

[24] Denny L, Kuhn L, Pollack A, Wright TC Jr. Direct visual inspection for cervical cancer Screening: an analysis of factors influencing test performance. Cancer 2002; 94,1699-707.

[25] Megevand E., Denny L, Dehaeck, K., Soeters, R, Bloch, B. Acetic acid visualization of the cervix: an alternative to cytologic screening. Obstet Gynecol 2001; 88(3), 383-386.

[26] Slawson D, Bennett J, Herman, J. Are Papanicolaou smears enough? Acetic acid washes of the cervix as adjunctive therapy: a HARNET study. Journal of Family Practice 1992; 35(3): 271-277.

[27] Gravitt PE, Paul P, Katki HA, Vendantham H, Ramakrishna G, et al. Effectiveness of VIA, Pap, and HPV DNA Testing in a Cervical Cancer Screening Program in a Peri-Urban Community in Andhra Pradesh, India. PLoS ONE 2010; 5(10), e13711. doi:10.1371/journal.pone.0013711

[28] Murillo R, Gamboa, L J, Osorio, O E, Cendales, B.J. (2010). Cervical Cancer Screening Study Group. Cervical cancer screening with naked-eye visual inspection in Colombia. Int J Gynaecol Obstet. 2010; 109, 230-234.

[29] Nessa A, Hussain MA, Rahman JN, Rashid MH, Muwonge R, Sankaranarayanan R. Screening for cervical neoplasia in Bangladesh using visual inspection with acetic acid. Int J Gynaecol Obstet. 2010; 111(2):115-8.

[30] Qureshi S, Das V, Zahra F. Evaluation of visual inspection with acetic acid and Lugol's iodine as cervical cancer screening tools in a low-resource setting. Trop Doct. 2010;40(1):9-12.

[31] Rana, T, Zia A, Sher S, Tariq, S, Asghar, F. Comparative Evaluation of PAP Smear and Visual Inspection of Acetic Acid (VIA) in Cervical Cancer Screening Program in Lady Willingdon Hospital, Lahore. Special Edition Annals 2010; 16 (1), 104-106.

[32] Akinola, O I, Fabamwo A, Oshodi Y, Banjo A, Odusanya O, Gbadegesin A, Tayo A. Efficacy of visual inspection of the cervix using acetic acid in cervical cancer screening: A comparison with cervical cytology. $\mathrm{J}$ Obstet Gynecol 2007; 27(7),703 -705. 